\title{
A Durable Minimal Intervention Strategy to Reduce Benzodiazepine Use in a Primary Care Population
}

\author{
Stephen Davidson ${ }^{1, *}$, Christine Thomson ${ }^{2}$, Gordon Prescott ${ }^{3}$ \\ ${ }^{1}$ Forensic Psychiatry, Rowanbank Clinic, NHS Greater Glasgow \& Clyde, Glasgow, UK \\ ${ }^{2}$ General Practice, Linkwood Medical, NHS Grampian, Elgin, UK \\ ${ }^{3}$ Medical Statistics Team, Institute of Applied Health Sciences, University of Aberdeen, Aberdeen, UK
}

Background: Benzodiazepines are commonly prescribed drugs with approximately $10 \%$ of adults having used them in the past year. These drugs are clearly addictive, yet many patients are prescribed these for years, with longterm side effects. The present study aimed to investigate whether patients on repeat diazepam prescription had their prescription reviewed to reduce and to stop the repeat prescription wherever appropriate, and whether these changes were sustained at 24 months.

Methods: The present study used a minimal intervention strategy to reduce diazepam use in a semi-rural general practice. Patients with a current prescription for diazepam were invited to visit their general practitioner for a review. Dose reduction grids were formulated for each individual to facilitate a downward titration by $1 \mathrm{mg}$ each wk/ mo. Patients with psychiatric co-morbidity were also included. Interrupted time series methods were applied to the monthly data. The outcomes were evaluated at 12 and 24 months.

Results: Ninety-two patients had diazepam on repeat prescription with 87 (94.6\%) attending the review appointment. Twenty-seven patients (29.3\%) were under psychiatric review and were supported by the psychiatrist with a downward titration regime. At 24 months, 63 patients (81.8\% of the 77 still at the practice) had stopped or were in the process of stopping regular use of diazepam. A statistically significant reduction in total monthly diazepam prescription was observed (from 2.2 to 0.7 defined daily dose/1,000 patients/d).

Conclusion: This minimal intervention strategy, in collaboration between primary and secondary care, produced a durable reduction in overall diazepam prescription at the general practice.

Keywords: Primary Health Care; Psychiatry; Benzodiazepines; Diazepam

Received: September 12, 2018, Revised: October 6, 2018, Accepted: October 18, 2018

${ }^{*}$ Corresponding Author: Stephen Davidson https://orcid.org/0000-0002-4536-476X Tel: +44-141-211-6572, E-mail: s.davidson7@nhs.net

'Early data from this study has been provisionally presented as a poster at the Royal College of Psychiatrists' Faculty of Addictions Annual Meeting 2016. 


\section{INTRODUCTION}

Benzodiazepines, and particularly diazepam, are commonly prescribed drugs. A recent study has found that almost $10 \%$ of the adults had taken a benzodiazepine in the past year. ${ }^{1)}$ Benzodiazepines are used for the treatment of epilepsy, severe muscle spasm, and as premedicants. However, majority of the prescriptions are for their hypnotic and anxiolytic effects. They may also be used as adjuncts in the treatment of depression and schizophrenia, ${ }^{1)}$ albeit with scarce evidence. $^{2,3)}$

Although useful in the management of generalized anxiety disorder (GAD), ${ }^{4,5)}$ perhaps less so in comparison to alternatives when used as hypnotics, ${ }^{6,7)}$ these drugs are clearly addictive. Many patients continue to take them for years with unknown benefits and likely harms. ${ }^{8)}$

NICE (The National Institute for Health and Care Excellence) recommends the use of benzodiazepines as a short-term measure during crisis in GAD, and not routinely for longer than a month. ${ }^{2)}$ Repeat prescriptions should be avoided in patients with major personality disorders or a history of substance misuse. In the latter group, benzodiazepines are often misused alongside other substances, ${ }^{9,10)}$ and are commonly implicated in drug related deaths. ${ }^{11)}$

Long-term side effects of chronic benzodiazepine use are well documented. These include increased risk of falls and associated sequel$\mathrm{ae}^{12)}$ anterograde amnesia, ${ }^{13)}$ and adverse effects on driving performance. ${ }^{14)}$ Withdrawal symptoms can occur after 4-6 weeks, and at least a third of long-term users experience problems on dosage reduction or withdrawal. ${ }^{15)}$

The decision to prescribe benzodiazepines is complex and multifactorial with particular individual variation. ${ }^{16)}$ Unfortunately, given the reduced continuity of care within general practice, unmonitored and repeat benzodiazepine prescriptions are common. Long-term side effects are of particular concern among older adults. ${ }^{12,13)}$

In the United Kingdom, the majority of prescriptions in general practice are written by medical doctors, with some practices employing non-medical prescribers such as pharmacists and nurses, who have undergone additional postgraduate training. Diazepam, like most benzodiazepines and non-benzodiazepine hypnotics, is listed as a controlled drug under Misuse of Drugs Regulations 2001 (UK) under schedule 4, part 1. It can be prescribed by medical doctors and specified non-medical prescribers including nurses and pharmacists.

Linkwood Medical is a semi-rural general practice located in Elgin, United Kingdom with a practice population of 11,400. It is an established training practice for junior doctors and general practitioner (GP) trainees. It has seen a $60 \%$ increase in the number of diazepam prescriptions given to patients over the past 4 years after correction for practice population numbers. The defined daily dose (DDD) per 1,000 patients on the practice list per day increased from 1.60 to 2.52 during this period. This rise is associated with a wide variation in prescribing habits between individual GPs, and has prompted further investigation.

Minimal intervention strategies have been demonstrated to increase the odds of a patient stopping their benzodiazepine by threefold. ${ }^{17-20)}$ These odds are further doubled by creation of systematic reduction strategies. ${ }^{17,19)}$ This evidence was the basis for our methodology.

We had the following questions before the study. Did the patients on repeat diazepam prescription have their prescriptions reviewed? If reviewed by their own GP, could diazepam doses for the patients on repeat diazepam prescription be titrated down? What were the outcomes for patients at 12 and 24 months?

\section{METHODS}

\section{Study Design}

Formal planning for the study took place from October 2014, including identification of patients, with the full intervention taking place between January 2015 and January 2017. All the patients with "current" repeat prescription of diazepam (receiving it at regular intervals with/ without other long-term therapies) were identified on "Vision" (primary care IT system with electronic patient record and prescribing). The individual files were examined for indication, dose, treatment duration, and review details.

Patients were sent a specific review appointment letter according to the appointment capacity. This letter advised the patients about the importance of attending the review appointment to allow appropriate diazepam prescribing, to receive support, and to discuss any difficulties with their GP. Tapering regimes were formulated by the pharmacist prescriber or the medication technician based on current best practice. ${ }^{17,21)}$ Dose reduction grids for each patient facilitated a downward titration of $1 \mathrm{mg}$ each wk/mo depending upon the individual circumstances.

The pharmacy team determined the exact quantities and doses of tablets for the patient while liaising with the local dispensaries. All the prescriptions were dispensed weekly, based on current recommendations. $^{22)}$

\section{Selection of Study Subjects}

No exclusion criteria were applied while selecting the subjects. Problematic benzodiazepine withdrawal is expected in patients with a history of substance misuse and in those with a co-morbid severe physical/psychiatric disorder or personality disorder. ${ }^{15,23)}$ Complex patients were titrated down, where appropriate, by their psychiatrist after liaising with their GP, and were included in the results.

\section{Measurements and Outcomes}

Outcomes for deceased individuals or those who had moved practice were noted as a part of an attrition analysis. Evaluation was carried out at 12 months and 24 months from the beginning of the study to allow assessment of the initial effect of the intervention and relapse-prevention.

Monthly aggregated data from January 2012 to February 2017 were extracted from the PIS (Prescribing Information System) using the PRISMS (Prescribing Information System for Scotland) database 
which allows National Health Service (NHS, UK) staff access to prescription data. DDD per 1,000 patients per day of diazepam was selected as a technical, standardized unit to measure the trend in diazepam prescribing over time. This is an assumed average maintenance dose per day for a drug and does not necessarily represent true consumption. Data were also obtained for all benzodiazepines.

\section{Statistical Analysis}

Pre-intervention characteristics of patients with diazepam on repeat prescription within the last 6 months were described using counts and percentages. Given the temporal nature of the monthly rates of prescriptions of benzodiazepines, and the potential presence of secular trends, interrupted time series methodology was used. Two approaches were used to evaluate the change in the rates of prescriptions in DDDs per 1,000 patients per day. Both the approaches allowed first order autocorrelation-dependence between successive values. The first was an autoregressive integrated moving average (ARIMA) regression model, which allowed investigation of seasonal patterns alongside other parameters by including pairs of sine and cosine terms. This was

Table 1. Patient demographics (percentages omitted for counts less than 10) $(\mathrm{N}=92)$

\begin{tabular}{|c|c|c|}
\hline $\begin{array}{l}\text { Pre-intervention } \\
\text { characteristics }\end{array}$ & Category & No. of patients (\%) \\
\hline \multirow[t]{2}{*}{ Gender } & Male & $56(60.9)$ \\
\hline & Female & $36(39.1)$ \\
\hline \multirow[t]{6}{*}{ Age (y) } & 20-39 & $10(10.9)$ \\
\hline & $40-49$ & $16(17.4)$ \\
\hline & $50-59$ & $24(26.1)$ \\
\hline & $60-69$ & $20(21.7)$ \\
\hline & $70-79$ & $13(14.1)$ \\
\hline & $80-89$ & 9 \\
\hline \multirow[t]{4}{*}{ Duration of diazepam } & $<12 \mathrm{mo}$ & $14(15.2)$ \\
\hline & $12-36 \mathrm{mo}$ & $19(20.7)$ \\
\hline & $37-60 \mathrm{mo}$ & $18(19.6)$ \\
\hline & $>60 \mathrm{mo}$ & $41(44.6)$ \\
\hline \multirow[t]{5}{*}{ Daily dose of diazepam } & As required dosing & 8 \\
\hline & $<2.5 \mathrm{mg}$ & $22(23.9)$ \\
\hline & $2.5-5 \mathrm{mg}$ & $31(33.7)$ \\
\hline & $5.5-10 \mathrm{mg}$ & $19(20.7)$ \\
\hline & $>10 \mathrm{mg}$ & $12(13.0)$ \\
\hline \multirow[t]{7}{*}{ Indication for diazepam } & Depression or anxiety & $40(43.5)$ \\
\hline & Addiction & 2 \\
\hline & Other psychiatric & $15(16.3)$ \\
\hline & Musculoskeletal pain & $16(17.4)$ \\
\hline & Neurological conditions & 8 \\
\hline & Insomnia & 4 \\
\hline & Other & 7 \\
\hline \multirow[t]{3}{*}{ Last review } & $<4 \mathrm{mo}$ & $52(56.5)$ \\
\hline & $4-12$ mo & $27(29.3)$ \\
\hline & $>12 \mathrm{mo}$ & $13(14.1)$ \\
\hline \multirow[t]{3}{*}{ Clinician review } & General practitioner & $55(59.8)$ \\
\hline & Psychiatrist & $27(29.3)$ \\
\hline & Non-medical prescriber & $10(10.9)$ \\
\hline \multirow[t]{3}{*}{ Psychiatry involvement } & Yes & 35 (38.0) \\
\hline & No & $50(54.3)$ \\
\hline & Previous & 7 \\
\hline
\end{tabular}

followed by an interrupted time series analysis (ITSA) with Prais-Winsten regression model through ITSA in Stata (statistical software package). ${ }^{24)}$ This method fits a linear regression line through monthly observations before an intervention and allows a change of intercept at intervention (a step up or down in the estimate). It also allows a change of slope after the intervention (an entirely different estimated change per month). The method enables multiple intervention times to represent a change of intercept and slope at the start of the intervention (October 2014) and further changes in both at the full implementation (January 2015). It also provides estimates, with confidence intervals, of the various intercepts and slopes but has limitations pertaining to inclusion of other structures such as seasonality.

\section{Ethical Approval}

This study was considered a local service evaluation or a quality improvement project following review of the proposal by the NHS Grampian Clinical Effectiveness Team. This team is responsible for the registration of all audit and service evaluation projects that are driven by individual clinicians across NHS Grampian. The project was registered with their database (ID: 4058). Therefore, ethical approval was not required.

\section{RESULTS}

\section{Pre-intervention}

Ninety-two patients had a repeat prescription for diazepam. Sixty-one percent were male. Age of the patients ranged from 28 to 83 years. The average time on diazepam was 81 months (range, 2 months to 23 years). Forty-five percent of the patients had been on diazepam for more than 5 years. The total daily dose was between $2 \mathrm{mg}$ and $25 \mathrm{mg}$. Fifty-seven (62\%) of the repeat prescriptions of diazepam were initially prescribed for a psychiatric indication. Fifty-two patients (56.5\%) had undergone a medication review past 4 months.

Initially, 27 patients (29.3\%) were under psychiatric evaluation. Their diazepam dose was reviewed and reduced, if appropriate, by their psychiatrist. Eight patients (8.7\%) were occasionally using extremely low doses of diazepam and were given a suitable titration plan. Fifty-seven patients (62.0\%) were eligible for the standard intervention (Table 1).

\section{Post-intervention}

Initial follow-up took place 12 months after the intervention. Attendance was very high, with 87 patients (94.6\%) attending the review appointment to discuss the intervention. At 12 months, 51 patients (55.4\%) had successfully titrated down and stopped diazepam (Table 2). Two patients were continued on a slower titration plan, 11 (12.0\%) were using diazepam intermittently, and $28(30.4 \%)$ were unable to stop using diazepam. Due to a boundary change, seven patients had moved GP practice. Eighty patients remained at the practice. Five patients had died. In total, 28 patients (30.4\%) were unable to participate in a reducing regime. 
Table 2. Patient outcomes for intervention $(\mathrm{N}=92)$

\begin{tabular}{|c|c|c|c|c|c|c|c|c|}
\hline \multirow{2}{*}{ Outcome } & \multicolumn{4}{|c|}{12 Months (January 2016) } & \multicolumn{4}{|c|}{24 Months (January 2017) } \\
\hline & Total & Moved & Deceased & Still at practice & Total & Moved & Deceased & Still at practice \\
\hline Stopped completely & $51(55.4)$ & 2 & 2 & $47(51.1)$ & $58(63.0)$ & 4 & 2 & $52(56.5)$ \\
\hline On slower titration plan & 2 & & & 2 & 2 & & & 2 \\
\hline Intermittent use & $11(12.0)$ & & 1 & $10(10.9)$ & $11(12.0)$ & 1 & 1 & $9(9.8)$ \\
\hline Unable to stop & $28(30.4)$ & 5 & 2 & $21(22.8)$ & $21(22.8)$ & 5 & 2 & $14(15.2)$ \\
\hline Total & $92(100)$ & 7 & 5 & $80(87.0)$ & $92(100)$ & $10(10.8)$ & 5 & $77(83.7)$ \\
\hline
\end{tabular}

Values are presented as number (\%) or number.

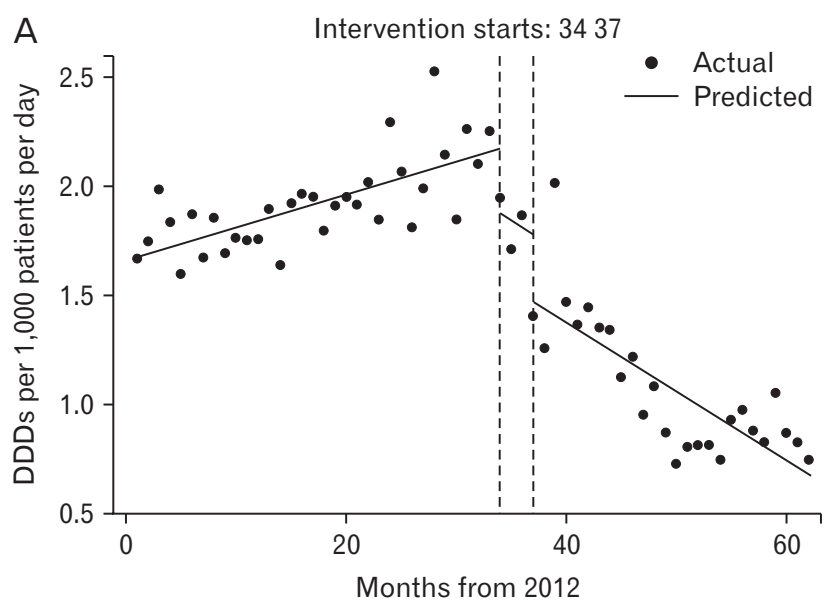

Prais-Winsten and Cochrane-Orcutt regression - lag (1)

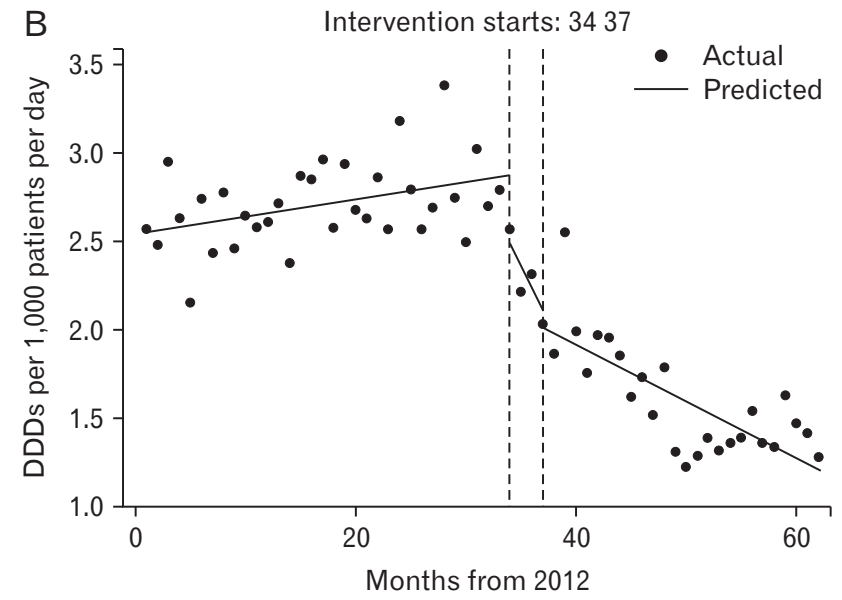

Prais-Winsten and Cochrane-Orcutt regression - lag (1)

Figure 1. Observed and predicted DDD per 1,000 patients per day for diazepam (A) and benzodiazepine (B) with change points in October 2014 and January 2015 (months 34 and 37). DDD, defined daily dose.

At 24 months since the beginning of the intervention, 58 patients (63.0\% of the 92 and $75.3 \%$ of the 77 still at the practice) had successfully titrated down and stopped diazepam. Nine of these patients had been given a one-off acute prescription for a specific purpose. Two patients continued with a slower titration plan, one under practice care, and the other by psychiatry. During 2016, 3 more patients moved practice. Sixty-three patients ( $81.8 \%$ of the 77 still at the practice) had stopped or were in the process of stopping regular use of diazepam. Out of 14 patients ( $18.2 \%$ of the 77 patients still at the practice) unable to stop regular use of diazepam, three patients were under care for learning disabilities and five were under care of psychiatry.

The ARIMA time series analysis showed no evidence of any seasonal effects and these were removed from the models. The ITSA model was applied with two points of change of intercept and slope at months 34 (October 2014, at the start of the intervention) and 37 (January 2015, at full implementation). For diazepam, the estimated prescribing rate was 1.7 DDD per 1,000 patients per day per month (pp$\mathrm{dpm}$ ), increasing by 0.015 per month ( $95 \%$ confidence interval [CI], 0.008 to 0.022 ), to approximately $2.2 \mathrm{DDD}$ per 1,000 ppdpm in October 2014 (month 34). There were two substantial but non-significant step decreases of around $0.3 \mathrm{DDD}$ per 1,000 ppdpm, at the start of the intervention (month 34), and at full implementation (month 37). There was a non-significant rate of decline of 0.032 DDD per 1,000 ppdpm
(95\% CI, -0.210 to 0.273$)$ during implementation. Following full implementation at month 37 (January 2015), the prescribing rate was 1.5 DDD per 1,000 ppdpm with a statistically significant linear rate of decline of 0.032 per month ( $95 \% \mathrm{CI}, 0.022$ to 0.042 ). By the end of the study, the prescribing rate was approximately 0.7 DDD per $1,000 \mathrm{pp}$ dpm (Figure 1A).

For benzodiazepines (Figure 1B), the estimated initial prescribing rate was $2.6 \mathrm{DDD}$ per 1,000 ppdpm, increasing by 0.010 per month (95\% CI, 0.002 to 0.017 ) before the intervention, to approximately 2.8 DDD per 1,000 ppdpm at month 34 . At this point, there was a large, non-significant step decrease of 0.390 (95\% CI, -0.026 to 0.806 ) and a steep decline by 0.126 DDD per 1,000 ppdpm ( $95 \%$ CI, -0.180 to 0.432 ). Following full implementation at month 37 , the prescribing rate was around 2.0 DDD per 1,000 ppdpm with a shallower decline of 0.032 DDD per 1,000 ppdpm ( $95 \%$ CI, 0.021 to 0.043 ) to a rate of $1.3 \mathrm{DDD}$ per 1,000 ppdpm by the end of the study. The monthly linear declines in diazepam and benzodiazepines prescribing rates were almost identical after full implementation at month 37.

\section{DISCUSSION}

\section{Summary}

At 24 months, the vast majority of patients who were previously on re- 
peat diazepam prescriptions were completely off the medication, with a smaller proportion using diazepam on an intermittent and specific basis.

\section{Strengths and Limitations}

The success of this intervention can be attributed to many factors, all involving multidisciplinary teamwork including the planning of appointments, advance preparation of individual diazepam dosing charts and prescriptions, documentation of stages in the notes, and monitoring of patient attendance. The project depended on shared understanding from the GPs and the pharmacy team.

Individual circumstances were considered before agreeing on downward titration plans. We achieved good concordance and patients were strongly encouraged to stay on plan, rather than reduce the dose more rapidly than the recommended regimen. Patients on longstanding diazepam prescriptions were given a monthly dose reduction and those on shorter term or smaller doses, a weekly dose reduction. It was considered important that patients were scheduled with their usual GP.

An effective working relationship with the local psychiatrist, for advice and discussion, was advantageous for more complex patients. This included weekly clinics to consult with patients, and a quarterly meeting with clinicians at the practice to discuss patients' progress. The psychiatrist established and concurred the plans with their patients who were able to reduce their diazepam.

The present study had some limitations. A gold standard follow-up investigation could have included repeat urine drug screens on completion of the titration regime. This would have confirmed that patients were not sourcing diazepam from alternative means. This was difficult to justify in a group that was using diazepam regularly but was not likely to meet diagnostic criteria for benzodiazepine dependence. ${ }^{25)}$

Diazepam was the only benzodiazepine examined separately. A similar study of other commonly prescribed benzodiazepines could have been conducted. However, it would have involved far fewer patients. Figure 1B demonstrates that there was no compensatory pattern of prescribing alternative benzodiazepines while diazepam prescribing had reduced.

Ten patients (10.9\%) had moved to neighboring GP practices due to a newly enforced boundary change. The change in practice policy of prescribing diazepam was not a factor for patients moving to other practices as confirmed by our attrition analysis.

\section{Comparison with Existing Literature}

The findings in the present study are consistent with those of previous studies on the successful use of minimal intervention strategies in primary care. ${ }^{17-20)}$ In contrast to similar studies, ${ }^{19,20)}$ our coordination with the local psychiatrist allowed the investigation of the intervention on patients involved with psychiatry and substance misuse services.

There has been limited research into relapse prevention following such interventions with the exception of two studies. In comparison to the study by Voshaar et al., ${ }^{26)}$ the present study was more proactive by ensuring all the patients at the practice had an attempted reduction, rather than a patient-led opt-in with comparatively favorable results. Vicens et al. ${ }^{27)}$ found that an intense follow-up regime was demonstrated similar efficacy to a structured intervention with written instructions, which supports the decision to use the latter approach in our study. Both the studies excluded psychiatric patients.

\section{Implications for Research and/or Practice}

New patients to this practice, who are on repeat prescriptions of diazepam, are immediately scheduled for an appointment with a GP to review their diazepam prescription. For patients authorized to use diazepam on an as required basis, the medical files are clearly annotated with the amount and frequency of the authorized dose. It is practice policy for diazepam to be dispensed weekly for all patients. These are important long-term safeguards to ensure that a patient's use of the drug does not change without good reason or review. This should be considered a gold standard for General Practice in prescribing diazepam and other drugs with a propensity for causing iatrogenic dependence.

Future challenges remain, with arguably the most complex patients continuing to receive long-term diazepam prescriptions, which are advised by their secondary care provider (psychiatry, learning disabilities or substance misuse services). In these cases, there is an evidence base supporting long-term benzodiazepine use. ${ }^{28)}$ A collaborative dialogue between primary and secondary care is imperative for reducing the number of patients remaining on diazepam prescriptions when not clinically indicated.

There is growing evidence for encouraging approaches that modify GP attitudes towards prescribing benzodiazepines and utilizing nonpharmacological alternatives. ${ }^{29,30)}$ Maintenance of positive prescribing practices and associated attitudes between GP partners has been streamlined through practice meetings and a practice protocol for the prescribing of diazepam. This information has been added to the practice's induction program and locum pack. This could be implemented in all similar teaching practices.

\section{Conclusion}

To summarize, this study has been able to produce a statistically significant, durable reduction in overall diazepam prescribing by using a minimal intervention strategy and maintaining a collaborative, proactive relationship between primary and secondary care providers.

\section{CONFLICT OF INTEREST}

No potential conflict of interest relevant to this article was reported.

\section{ACKNOWLEDGMENTS}

The authors would like to thank the pharmacy team at Linkwood Medical, the general practitioners, and the local psychiatrist for their support in implementing this minimal intervention in a safe and com- 
passionate manner. The authors would like to recognize the role of Karen Box in collating the prescribing data. Finally, the authors would like to thank the patients involved in the study, with many having made a responsible, informed choice to reduce or stop their regular diazepam prescriptions when this medication was not absolutely necessary.

\section{ORCID}

Stephen Davidson: https://orcid.org/0000-0002-4536-476X

Gordon Prescott: https://orcid.org/0000-0002-9156-2361

\section{REFERENCES}

1. Demyttenaere K, Bonnewyn A, Bruffaerts R, De Girolamo G, Gasquet I, Kovess V, et al. Clinical factors influencing the prescription of antidepressants and benzodiazepines: results from the European study of the epidemiology of mental disorders (ESEMeD). J Affect Disord 2008;110:84-93.

2. National Institute for Health and Clinical Excellence. Generalised anxiety disorder and panic disorder (with or without agoraphobia) in adults: clinical guideline CG113 [Internet]. London: National Institute for Health and Clinical Excellence; 2011 [cited 2016 Oct 10]. Available from: https://www.nice.org.uk.

3. Volz A, Khorsand V, Gillies D, Leucht S. Benzodiazepines for schizophrenia. Cochrane Database Syst Rev 2007:CD006391.

4. Martin JL, Sainz-Pardo M, Furukawa TA, Martin-Sanchez E, Seoane T, Galan C. Benzodiazepines in generalized anxiety disorder: heterogeneity of outcomes based on a systematic review and meta-analysis of clinical trials. J Psychopharmacol 2007;21:774-82.

5. Davidson JR, Zhang W, Connor KM, Ji J, Jobson K, Lecrubier Y, et al. A psychopharmacological treatment algorithm for generalised anxiety disorder (GAD). J Psychopharmacol 2010;24:3-26.

6. Krystal AD, Erman M, Zammit GK, Soubrane C, Roth T; ZOLONG Study Group. Long-term efficacy and safety of zolpidem extended-release $12.5 \mathrm{mg}$, administered 3 to 7 nights per week for 24 weeks, in patients with chronic primary insomnia: a 6-month, randomized, double-blind, placebo-controlled, parallel-group, multicenter study. Sleep 2008;31:79-90.

7. Krystal AD, Walsh JK, Laska E, Caron J, Amato DA, Wessel TC, et al. Sustained efficacy of eszopiclone over 6 months of nightly treatment: results of a randomized, double-blind, placebo-controlled study in adults with chronic insomnia. Sleep 2003;26:793-9.

8. Benitez CI, Smith K, Vasile RG, Rende R, Edelen MO, Keller MB. Use of benzodiazepines and selective serotonin reuptake inhibitors in middle-aged and older adults with anxiety disorders: a longitudinal and prospective study. Am J Geriatr Psychiatry 2008;16:5-13.

9. National Collaborating Centre for Mental Health (UK). Drug misuse: opioid detoxification. Leicester: British Psychological Society; 2008.

10. Hopley AA, Brunelle C. Personality mediators of psychopathy and substance dependence in male offenders. Addict Behav 2012;37:94755.

11. Department of Health (England); Devolved Administrations. Drug misuse and dependence: UK guidelines on clinical management.
London: National Treatment Agency for Substance Misuse; 2007.

12. Wang PS, Bohn RL, Glynn RJ, Mogun H, Avorn J. Hazardous benzodiazepine regimens in the elderly: effects of half-life, dosage, and duration on risk of hip fracture. Am J Psychiatry 2001;158:892-8.

13. Verwey B, Eling P, Wientjes H, Zitman FG. Memory impairment in those who attempted suicide by benzodiazepine overdose. J Clin Psychiatry 2000;61:456-9.

14. Barbone F, McMahon AD, Davey PG, Morris AD, Reid IC, McDevitt DG, et al. Association of road-traffic accidents with benzodiazepine use. Lancet 1998;352:1331-6.

15. Schweizer E, Rickels K. Benzodiazepine dependence and withdrawal: a review of the syndrome and its clinical management. Acta Psychiatr Scand Suppl 1998;393:95-101.

16. Sirdifield C, Anthierens S, Creupelandt H, Chipchase SY, Christiaens T, Siriwardena AN. General practitioners' experiences and perceptions of benzodiazepine prescribing: systematic review and meta-synthesis. BMC Fam Pract 2013;14:191.

17. Voshaar RC, Couvee JE, van Balkom AJ, Mulder PG, Zitman FG. Strategies for discontinuing long-term benzodiazepine use: meta-analysis. Br J Psychiatry 2006;189:213-20.

18. Salonoja M, Salminen M, Aarnio P, Vahlberg T, Kivela SL. One-time counselling decreases the use of benzodiazepines and related drugs among community-dwelling older persons. Age Ageing 2010;39:313-9.

19. Vicens C, Bejarano F, Sempere E, Mateu C, Fiol F, Socias I, et al. Comparative efficacy of two interventions to discontinue long-term benzodiazepine use: cluster randomised controlled trial in primary care. $\mathrm{Br} \mathrm{J}$ Psychiatry 2014;204:471-9.

20. Lopez-Peig C, Mundet X, Casabella B, del Val JL, Lacasta D, Diogene E. Analysis of benzodiazepine withdrawal program managed by primary care nurses in Spain. BMC Res Notes 2012;5:684.

21. Denis C, Fatseas M, Lavie E, Auriacombe M. Pharmacological interventions for benzodiazepine mono-dependence management in outpatient settings. Cochrane Database Syst Rev 2006:CD005194.

22. Parr JM, Kavanagh DJ, Cahill L, Mitchell G, McD Young R. Effectiveness of current treatment approaches for benzodiazepine discontinuation: a meta-analysis. Addiction 2009;104:13-24.

23. Tyrer P. Risks of dependence on benzodiazepine drugs: the importance of patient selection. BMJ 1989;298:102, 104-5.

24. StataCorp. Stata statistical software: release 14. College Station (TX): StataCorp LP; 2015.

25. World Health Organization. The ICD-10 classification of mental and behavioural disorders: clinical descriptions and diagnostic guidelines. Geneva: World Health Organization; 1992.

26. Voshaar RO, Gorgels W, Mol A, van Balkom A, Breteler M, van de Lisdonk E, et al. Predictors of relapse after discontinuation of long-term benzodiazepine use by minimal intervention: a 2-year follow-up study. Fam Pract 2003;20:370-2.

27. Vicens C, Sempere E, Bejarano F, Socias I, Mateu C, Fiol F, et al. Efficacy of two interventions on the discontinuation of benzodiazepines in long-term users: 36-month follow-up of a cluster randomised trial in primary care. Br J Gen Pract 2016;66:e85-91.

28. Baldwin DS, Aitchison K, Bateson A, Curran HV, Davies S, Leonard B, et al. Benzodiazepines: risks and benefits: a reconsideration. J Psychopharmacol 2013;27:967-71.

29. Creupelandt H, Anthierens S, Habraken H, Declercq T, Sirdifield C, 
Siriwardena AN, et al. Teaching young GPs to cope with psychosocial consultations without prescribing: a durable impact of an e-module on determinants of benzodiazepines prescribing. BMC Med Educ 2017;17:259.
30. Pottie K, Thompson W, Davies S, Grenier J, Sadowski CA, Welch V, et al. Deprescribing benzodiazepine receptor agonists: Evidence-based clinical practice guideline. Can Fam Physician 2018;64:339-51. 